\title{
ONE-POT SYNTHESIS OF SPIRO-3,4-DIHYDRO-2H- PYRROLES THROUGH TANDEM NUCLEOPHILIC CYCLISATION REACTION
}

\author{
Nandkishor Chandan \\ Department of Chemistry, Siddharth College of Arts, Science and Commerce, \\ Fort Mumbai-400 001, Maharashtra, INDIA. \\ ${ }^{\square}$ Corresponding Author: nandkishorc@gmail.com
}

\begin{abstract}
A very simple and convenient one-pot synthesis of Spiro-3,4-dihydro- $2 H$-pyrrole has been developed while synthesizing 2,2,5-trisubstituted pyrrolidines. Initially, the Meldrum's acid was treated with $\alpha, \beta$-unsaturated ketones in presence of anhydrous carbonate base and phase transfer catalyst benzyltriethylammonium chloride in acetonitrile as a solvent to afforded the Michael adduct which was readily converted to the corresponding oxime using standard conditions. The crude oxime was treated directly with $p$-toluenesulfonyl chloride to tosylate the oxime but instead in presence of an excess of organic base as $\mathrm{Et}_{3} \mathrm{~N}$ results in tandem nucleophilic cyclization to spirobicyclic compound as Spiro-3,4-dihydro- $2 H$-pyrrole as product directly, instead of the tosyloxime. So, it was considered to be a novel route to synthesized spirocyclic compounds as it is the skeleton of many natural products and pharmaceutical products.
\end{abstract}

Keywords: Pyrrole, Spiro, Oxime, Tosyloxime, Cyclization, Meldrum's Acid, Tandem, Nucleophilic, $\alpha, \beta-$ Unsaturated Ketone.

RASĀYAN J. Chem., Vol. 14, No.2, 2021

\section{INTRODUCTION}

The substituted Pyrroles are the most important and explored heterocyclic compounds as they are found in many natural products and biologically active compounds ${ }^{1-3}$ as well are privileged scaffolds for pharmaceutical products ${ }^{4-8}$ since they exhibit a diverse range of biological activities, including analgesic, anti-convulsant, anti-depressant, anti-allergic, anti-diabetic, anti-hyperlipidemic, anti-microbial, antifungal, anti-viral, anti-inflammatory, cholesterol-reducing, and anti-tumor properties. ${ }^{9-12}$ Therefore, substituted pyrroles are considered as a potential source of biologically active compounds with valuable properties.

The Spiro heterocyclic compounds are considered as a privileged framework because of their rigidity, three-dimensional geometries, and wide distribution in various natural products and synthetic molecules. Presently, the Spiro-heterobicyclic compounds are attracting considerable interest in synthetic organic chemistry because of their molecular structure and diverse biological activities ${ }^{13}$. In addition, spiroheterocycle systems impart structural novelty for drug discovery because of its broad range of medicinal properties, ${ }^{14-18}$ as well important structural motifs that are entrenched in a variety of drugs, including the cardiovascular active Irbesartan, ${ }^{19-20}$ progestogenic agent Drospirenone ${ }^{21-22}$ and potassium-separating diuretic Spironolactone. ${ }^{23-24}$ The normally used approach in drug designing is to rigidify the ligand conformation by a spiro-ring fusion, which can impact ligand binding entropy upon binding to a protein target. Commonly, bioactive spirocyclic molecules have five-membered ring systems in their structures. In particular, spiro-pyrroles represent important structural motifs that can be found in many biologically active synthetic compounds and natural products. ${ }^{25-26} \mathrm{We}$ have recently reported that, under basic conditions, tosyloxime of Michael adduct with diethyl malonate results in the cyclization with $\mathrm{C}-\mathrm{N}$ bond formation to pyrrolidine embedded with various functionality ${ }^{27-28}$. In a similar line to introduced the rigidity, we have used Meldrum's acid as a source of nucleophile in Michael addition reaction with different $\alpha, \beta$-unsaturated ketones which on oximation and followed by tosylation more than basic condition results into the Spiro-3,4-dihydro-2H-pyrrole in good yield as one-pot synthesis (Fig.-1).

Rasayan J. Chem., 14(2), 974-980(2021) 
RASĀYAN J. Chem.

Vol. 14 | No. 2 |974-980| April - June | 2021
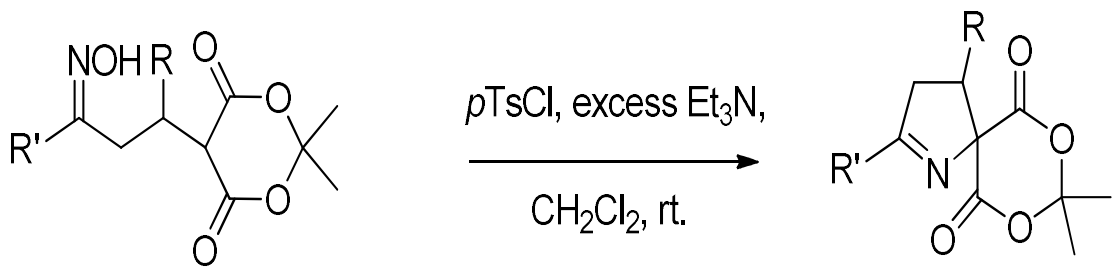

Fig.-1: Cyclization Leads to Spiro Compound

\section{EXPERIMENTAL \\ General Procedure for the Preparation of Michael Adduct ${ }^{9-12}$}

To a stirring solution, $7.63 \mathrm{mmol}$ of Meldrumic acid $(1.1 \mathrm{~g})$ in acetonitrile $(20 \mathrm{ml})$ was added $8.39 \mathrm{mmol}$ of anhydrous $\mathrm{K}_{2} \mathrm{CO}_{3}(1.15 \mathrm{~g})$ and $7.63 \mathrm{mmol}$ of benzyltriethylammonium chloride $(1.73 \mathrm{~g})$. The mixture was stirred for $15 \mathrm{~min}$ at $\mathrm{rt}$ and $7.63 \mathrm{mmol}$ of methyl vinyl ketone $(0.64 \mathrm{ml})$ was then added and the resultant mixture was stirred for $8 \mathrm{hr}$ at $50^{\circ} \mathrm{C}$. After TLC was monitored, the mixture was cooled to $\mathrm{rt}$ and the reaction was quenched with water and mixture was washed with diethyl ether, then acidified by $6 \mathrm{~N}$ $\mathrm{HCl}$. The adduct was extracted by diethyl ether and dried over $\mathrm{Na}_{2} \mathrm{SO}_{4}$, to give the crude product, which was purified by recrystallization using EtOAc and petroleum ether to afford Michael adduct $\mathbf{1}$.

\section{General Procedure for the Preparation Oxime of Michael Adduct ${ }^{27-28}$}

To the solution of $2.33 \mathrm{mmol}$ Michael adduct ketone $1(500 \mathrm{mg})$ in $10 \mathrm{ml} \mathrm{EtOH}$ was added $4.66 \mathrm{mmol}$ of $\mathrm{NH}_{2} \mathrm{OH} \cdot \mathrm{HCl}(183 \mathrm{mg})$ and $4.66 \mathrm{mmol}$ of $\mathrm{Et}_{3} \mathrm{~N}(470 \mathrm{mg})$ and the reaction mixture was heated to $50^{\circ \mathrm{C}}$ for 1 $\mathrm{hr}$. The progress of the reaction was monitored by TLC and on completion of the reaction mixture was allowed to cool to room temperature. EtOH was removed under reduced pressure and the residue was dissolved in EtOAc and $\mathrm{H}_{2} \mathrm{O}$ added. The organic layer was separated, washed with brine and dried over $\mathrm{Na}_{2} \mathrm{SO}_{4}$. The concentration of the organic layer gave the crude product, which was purified by flash column chromatography (eluting with EtOAc : petrol) to afford the oxime product 2.

\section{General Procedure for Cyclization to Spiro-3,4-dihydro-2H-pyrrole ${ }^{29}$}

The stirring solution $2.18 \mathrm{mmol}$ of 2 oxime $(500 \mathrm{mg})$ in dry $10 \mathrm{ml} \mathrm{CH}_{2} \mathrm{Cl}_{2}$ at $0{ }^{\circ} \mathrm{C}$ was treated with 6.54 mmol of $\mathrm{Et}_{3} \mathrm{~N}$ or pyridine $(660 \mathrm{mg}$ or $516 \mathrm{mg}$ ) followed by slow addition of $2.61 \mathrm{mmol}$ of $p$ toluenesulphonyl chloride (494mg) and the reaction was stirred for $1 \mathrm{hrs}$ and it was found in mass spectra showed the presence of the cyclized product. The reaction mixture was further stirred for $2 \mathrm{hrs}$ and the reaction progress was monitored by TLC and by mass spectra and it was found that instead of tosyloxime the cyclized product was formed and after completion of the reaction $(1 \mathrm{M}) \mathrm{HCl}$ was added and the product was extracted by $\mathrm{CH}_{2} \mathrm{Cl}_{2}$ and the extracts were washed with saturated $\mathrm{NaHCO}_{3}$ and dried over $\mathrm{Na}_{2} \mathrm{SO}_{4}$. The concentration of $\mathrm{CH}_{2} \mathrm{Cl}_{2}$ gave the crude product which was purified by flash column chromatography (eluting with EtOAc : petrol) to afford the Spiro-3,4-dihydro-2H-pyrrole.

( \pm ) Dimethyl-5-(3-oxobutyl)-1,3-dioxane-4,6-dione, $1^{32}$

Following the general procedure 1, to the stirring solution $15.3 \mathrm{mmol}$ of Meldrumic acid $(2.21 \mathrm{~g})$ in acetonitrile $(40 \mathrm{ml})$ was added $16.8 \mathrm{mmol}$ of anhydrous $\mathrm{K}_{2} \mathrm{CO}_{3}(2.31 \mathrm{~g})$ and $15.3 \mathrm{mmol}$ of benzyltriethylammonium chloride (3.48g). The mixture was stirred for $15 \mathrm{~min}$ at $\mathrm{rt}$ and $15.3 \mathrm{mmol}$ of methyl vinyl ketone $(1.3 \mathrm{ml})$ was then added and the resultant mixture was stirred for $8 \mathrm{hr}$ at $50{ }^{\circ} \mathrm{C}$ to give crude product, which was purified by recrystallization using EtOAc and petroleum ether to afford adduct $1(2.67 \mathrm{~g}, 81 \%)$ as pale yellow solid (m.p. $=116-118{ }^{\circ} \mathrm{C}$, lit $\left.119-120{ }^{\circ} \mathrm{C}\right)^{99} ; \mathrm{R}_{f}=0.16($ EtOAc : petrol, $1: 1) ; v_{\max }(\mathrm{film}) / \mathrm{cm}^{-1} 2892,1785,1747,1710,1382,1303,1249,1165,1010,986,730 ; \delta_{\mathrm{H}}(400 \mathrm{MHz} ;$ $\left.\mathrm{CDCl}_{3} ; \mathrm{Me}_{4} \mathrm{Si}\right) 1.74,1.78\left(6 \mathrm{H}, \mathrm{s}, \mathrm{C}\left(\mathrm{CH}_{3}\right)_{2}\right), 2.13\left(3 \mathrm{H}, \mathrm{s}, \mathrm{COCH}_{3}\right), 2.78\left(2 \mathrm{H}, \mathrm{m}, \mathrm{CH}_{2} \mathrm{CH} \mathrm{H}_{2} \mathrm{CH}\right), 2.75(2 \mathrm{H}, \mathrm{t}$, $\left.J 7.1 \mathrm{~Hz}, \mathrm{CH}_{2} \mathrm{CH}_{2} \mathrm{CH}\right), 3.86\left(1 \mathrm{H}, \mathrm{t}, J 5.4 \mathrm{~Hz}, \mathrm{CH}_{2} \mathrm{CH}_{2} \mathrm{CH}\right) ; \delta_{\mathrm{C}}\left(100 \mathrm{MHz} ; \mathrm{CDCl}_{3} ; \mathrm{Me}_{4} \mathrm{Si}\right) 19.5$ $\left.\left(\mathrm{CH}_{2} \mathrm{CH}_{2} \mathrm{CH}\right), 25.8,27.9 \mathrm{C}\left(\mathrm{CH}_{3}\right)_{2}\right), 29.5\left(\mathrm{COCH}_{3}\right), 38.7\left(\mathrm{CH}_{2} \mathrm{CH}_{2} \mathrm{CH}\right), 44.0\left(\mathrm{CH}_{2} \mathrm{CH}_{2} \mathrm{CH}\right), 104.5$ $\left(C\left(\mathrm{CH}_{3}\right)_{2}\right), 164.7$ (2 x COO), $207.5(\mathrm{CO}) ; \mathrm{m} / \mathrm{z}\left(\mathrm{ESI}^{-}\right)$213, ([M-H]', 100\%), HRMS (ESI) $\mathrm{C}_{10} \mathrm{H}_{13} \mathrm{O}_{5}^{-}$([M$\left.\mathrm{H}]^{-}\right)$requires 213.0768; found 213.0762. 
RASĀYAN J. Chem.

Vol. 14 | No. 2 |974-980| April - June | 2021

( \pm ) 5-(3-(Hydroxyimino)butyl)-2,2-dimethyl-1,3-dioxane-4,6-dione, $\mathbf{2}^{27-28}$

Following the general procedure 2, to the solution $6.44 \mathrm{mmol}$ of Michael adduct $1(1.38 \mathrm{~g})$ in EtOH (30ml), $12.88 \mathrm{mmol}$ of $\mathrm{NH}_{2} \mathrm{OH} . \mathrm{HCl}\left(888 \mathrm{mg}\right.$,) and $12.88 \mathrm{mmol}$ of $\mathrm{Et}_{3} \mathrm{~N}(1.30 \mathrm{~g}$ ) was added and the mixture was gently heated $\left(50{ }^{\circ} \mathrm{C}\right)$ for $1 \mathrm{hrs}$, to give crude product which was purified by flash column chromatography to afford oxime $2(1.14 \mathrm{~g}, 77 \%)$ as pale yellow semi-solid; $\mathrm{R}_{f}=0.23$ (EtOAc : petrol, $1: 1) ; v_{\max }(\mathrm{film}) / \mathrm{cm}^{-1} 3319,2982,1786,1743,1715,1372,1307,1240,1176,1012$, 982, 731; (major isomer) $\delta_{\mathrm{H}}\left(400 \mathrm{MHz} ; \mathrm{CDCl}_{3} ; \mathrm{Me}_{4} \mathrm{Si}\right) 1.74,1.78\left(6 \mathrm{H}, \mathrm{s}, \mathrm{C}\left(\mathrm{CH}_{3}\right)_{2}\right), 1.94,1.91\left(3 \mathrm{H}, \mathrm{s}, \mathrm{CNOHCH}_{3}\right), 2.31$ $\left(2 \mathrm{H}, \mathrm{m}, \mathrm{CH}_{2} \mathrm{CH}_{2} \mathrm{CH}\right), 2.44,2.63\left(2 \mathrm{H}, \mathrm{t}, J 7.4 \mathrm{~Hz}, \mathrm{CH}_{2} \mathrm{CH}_{2} \mathrm{CH}\right), 3.68\left(1 \mathrm{H}, \mathrm{t}, J 6.8 \mathrm{~Hz}, \mathrm{CH}_{2} \mathrm{CH}_{2} \mathrm{CH}\right), 9.33$ $(1 \mathrm{H}, \mathrm{bs}, \mathrm{OH}) ; \delta_{\mathrm{C}}\left(100 \mathrm{MHz} ; \mathrm{CDCl}_{3} ; \mathrm{Me}_{4} \mathrm{Si}\right) 13.9,15.1\left(\mathrm{CNOHCH}_{3}\right), 21.3,22.1\left(\mathrm{CH}_{2} \mathrm{CH}_{2} \mathrm{CH}\right), 26.2,27.3$

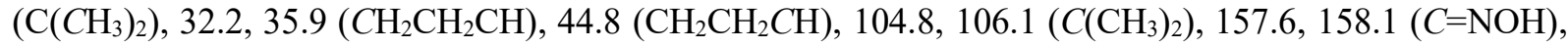
162.7, 164.9 (2 x COO); m/z (ESI) $228\left([\mathrm{M}-\mathrm{H}]^{-}, 100 \%\right)$, HRMS (ESI $)^{-} \mathrm{C}_{10} \mathrm{H}_{14} \mathrm{NO}_{5}{ }^{-}\left([\mathrm{M}-\mathrm{H}]^{-}\right)$requires 228.0881 ; found 228.0877 .

( \pm ) 2,8,8-Trimethyl-7,9-dioxa-1-azaspiro[4.5] dec-1-ene-6,10-dione, $3^{34-35}$

Following the general procedure 3, to the solution $1.79 \mathrm{mmol}$ of oxime $2(412 \mathrm{mg})$ in dry $\mathrm{CH}_{2} \mathrm{Cl}_{2}(20 \mathrm{ml})$ was added $5.37 \mathrm{mmol}$ of $\mathrm{Et}_{3} \mathrm{~N}(542 \mathrm{mg})$ at $0^{\circ} \mathrm{C}$ and followed by $2.15 \mathrm{~mol}$ of $p$-toluenesulphonyl chloride $(410 \mathrm{mg})$ and the mixture stirred for $30 \mathrm{~min}$ instead tosyl oxime, isolated cyclized product as spirobicyclic pyrrolidine which was purified by flash column chromatography to afford compound $\mathbf{3}(297 \mathrm{mg}, 78 \%)$ as a crystalline solid; m.p. $=98{ }^{\circ} \mathrm{C} ; \mathrm{R}_{f}=0.63$ (EtOAc : petrol, 1:1); $v_{\max }\left(\right.$ film) $/ \mathrm{cm}^{-1} 2933,1782,1731,1452$, $1170,914,731 ; \delta_{\mathrm{H}}\left(400 \mathrm{MHz} ; \mathrm{CDCl}_{3} ; \mathrm{Me}_{4} \mathrm{Si}\right) 1.78,2.06\left(\mathrm{C}\left(\mathrm{CH}_{3}\right)_{2}\right), 2.16\left(3 \mathrm{H}, \mathrm{s}, \mathrm{N}=\mathrm{CCH}_{3}\right), 2.59(2 \mathrm{H}, \mathrm{t}, J$ $7.6 \mathrm{~Hz}, \mathrm{C}(4) H H), 2.97(2 \mathrm{H}, \mathrm{t}, J 7.6 \mathrm{~Hz}, \mathrm{C}(3) H H) ; \delta_{\mathrm{C}}\left(100 \mathrm{MHz} ; \mathrm{CDCl}_{3} ; \mathrm{Me}_{4} \mathrm{Si}\right) 19.2\left(\mathrm{COCH}_{3}\right), 28.3,28.5$ $\left.\left.\mathrm{C}\left(\mathrm{CH}_{3}\right)_{2}\right), 31.4(\mathrm{C} 4), 41.2(\mathrm{C3}), 80.7(\mathrm{C} 2), 106.2 \mathrm{C}\left(\mathrm{CH}_{3}\right)_{2}\right), 167.4(2 \times \mathrm{COO}), 182.9(\mathrm{C} 5) ; \mathrm{m} / \mathrm{z}\left(\mathrm{ESI}^{+}\right) 445$ $\left([2 \mathrm{M}+\mathrm{Na}]^{+}, 100 \%\right), 234\left([\mathrm{M}+\mathrm{Na}]^{+}, 95 \%\right), \operatorname{HRMS}\left(\mathrm{ESI}^{+}\right) \mathrm{C}_{10} \mathrm{H}_{13} \mathrm{NNaO}_{5}{ }^{+}\left([\mathrm{M}+\mathrm{Na}]^{+}\right)$requires 234.0742 ; found 234.0737 .

\section{( \pm ) 4,8,8-trimethyl-2-phenyl-7,9-dioxa-1-azaspiro[4.5]dec-1-ene-6,10-dione, 6}

Following the general procedure $\mathbf{3}$, to the solution $0.95 \mathrm{mmol}$ of oxime $\mathbf{5}(289 \mathrm{mg})$ in dry $\mathrm{CH}_{2} \mathrm{Cl}_{2}(15 \mathrm{ml})$ was added $2.85 \mathrm{mmol}$ of $\mathrm{Et}_{3} \mathrm{~N}(288 \mathrm{mg})$ at $0^{\circ} \mathrm{C}$ and followed by $1.14 \mathrm{mmol}$ of $p$-toluenesulphonyl chloride $(216 \mathrm{mg}$ ) and the mixture was stirred for $30 \mathrm{~min}$ instead tosyl oxime, isolated cyclized product as spirobicyclic pyrrolidine which was purified by flash column chromatography to afford compound 6 (160 $\mathrm{mg}, 59 \%)$ as a crystalline solid; m.p. $=103{ }^{\circ} \mathrm{C} ; \mathrm{R}_{f}=0.46($ EtOAc : petrol, $1: 1.5) ; v_{\max }($ film $) / \mathrm{cm}^{-1} 3078$, 2956, 1779, 1737, 1170, 912, 740; $\delta_{\mathrm{H}}\left(400 \mathrm{MHz} ; \mathrm{CDCl}_{3} ; \mathrm{Me}_{4} \mathrm{Si}\right) 0.96\left(3 \mathrm{H}, \mathrm{d}, \mathrm{CH}_{3}\right), 1.75,2.00\left(\mathrm{C}\left(\mathrm{CH}_{3}\right)_{2}\right)$, $2.55(2 \mathrm{H}, \mathrm{d}, J 7.5 \mathrm{~Hz}, \mathrm{C}(4) H H), 2.79(1 \mathrm{H}, \mathrm{m}, \mathrm{C}(3) H), 7.3-7.9(\mathrm{Ar} H) ; \delta_{\mathrm{C}}\left(100 \mathrm{MHz} ; \mathrm{CDCl}_{3} ; \mathrm{Me}_{4} \mathrm{Si}\right) 28.9$, $\left.\left.28.2 \mathrm{C}\left(\mathrm{CH}_{3}\right)_{2}\right), 31.9(\mathrm{C} 4), 41.0(\mathrm{C3}), 89.7(\mathrm{C} 5), 106.0 \mathrm{C}\left(\mathrm{CH}_{3}\right)_{2}\right), 112-139(\mathrm{ArC}), 167.0(2 \times \mathrm{COO}), 180.9$ $(C 2) ; \mathrm{m} / \mathrm{z}\left(\mathrm{ESI}^{+}\right) 310\left([\mathrm{M}+\mathrm{Na}]^{+}, 95 \%\right), \mathrm{HRMS}\left(\mathrm{ESI}^{+}\right) \mathrm{C}_{16} \mathrm{H}_{17} \mathrm{NNaO}_{4}{ }^{+}\left([\mathrm{M}+\mathrm{Na}]^{+}\right)$requires 310.1055 ; found 234.1076.

( \pm ) 8,8-dimethyl-2-phenyl-7,9-dioxa-1-azaspiro[4.5]dec-1-ene-6,10-dione, 9

Following the general procedure 3, to the solution $0.69 \mathrm{mmol}$ of oxime $8(200 \mathrm{mg})$ in dry $\mathrm{CH}_{2} \mathrm{Cl}_{2}(10 \mathrm{ml})$ was added $2.07 \mathrm{mmol}$ of $\mathrm{Et}_{3} \mathrm{~N}(209 \mathrm{mg})$ at $0^{\circ} \mathrm{C}$ and followed by $0.83 \mathrm{mmol}$ of $p$-toluenesulphonyl chloride (157 mg,) and mixture was stirred for $30 \mathrm{~min}$ instead tosyl oxime, isolated cyclized product as spirobicyclic pyrrolidine which was purified by flash column chromatography to afford compound 9 (120 $\mathrm{mg}, 64 \%$ ) as a crystalline solid; m.p. $=97^{\circ} \mathrm{C} ; \mathrm{R}_{f}=0.53$ (EtOAc : petrol, 1:1); $v_{\max }\left(\right.$ film) $/ \mathrm{cm}^{-1} 3093,2931$, $1778,1735,1177,910 ; \delta_{\mathrm{H}}\left(400 \mathrm{MHz} ; \mathrm{CDCl}_{3} ; \mathrm{Me}_{4} \mathrm{Si}\right) 1.70,2.03\left(\mathrm{C}\left(\mathrm{CH}_{3}\right)_{2}\right), 2.52(2 \mathrm{H}, \mathrm{t}, J 7.4 \mathrm{~Hz}$, $\mathrm{C}(4) H H), 2.90(2 \mathrm{H}, \mathrm{t}, J 7.4 \mathrm{~Hz}, \mathrm{C}(3) H H), 7.3-7.9(\mathrm{Ar} H)$; $\delta_{\mathrm{C}}\left(100 \mathrm{MHz} ; \mathrm{CDCl}_{3} ; \mathrm{Me}_{4} \mathrm{Si}\right) 28.9,28.2$ $\left.\left.\mathrm{C}\left(\mathrm{CH}_{3}\right)_{2}\right), 31.9(\mathrm{C} 4), 41.0(\mathrm{C3}), 89.7(\mathrm{C5}), 106.0 \mathrm{C}\left(\mathrm{CH}_{3}\right)_{2}\right), 114-138(\mathrm{ArC}), 167.0$ (2 x COO), $180.9(\mathrm{C} 2)$; $\mathrm{m} / \mathrm{z}\left(\mathrm{ESI}^{+}\right) 296\left([\mathrm{M}+\mathrm{Na}]^{+}, 95 \%\right), \mathrm{HRMS}\left(\mathrm{ESI}^{+}\right) \mathrm{C}_{15} \mathrm{H}_{15} \mathrm{NNaO}_{4}{ }^{+}\left([\mathrm{M}+\mathrm{Na}]^{+}\right)$requires 296.0899; found 234.0901.

\section{( \pm ) 2,4,8,8-tetramethyl-7,9-dioxa-1-azaspiro[4.5]dec-1-ene-6,10-dione, 12}

Following the general procedure $\mathbf{3}$, to the solution $0.88 \mathrm{mmol}$ of oxime $\mathbf{1 1}(215 \mathrm{mg})$ in dry $\mathrm{CH}_{2} \mathrm{Cl}_{2}(10 \mathrm{ml})$ was added $2.64 \mathrm{mmol}$ of $\mathrm{Et}_{3} \mathrm{~N}(267 \mathrm{mg})$ at $0^{\circ} \mathrm{C}$ and followed by $1.06 \mathrm{mmol}$ of $p$-toluenesulphonyl 
RASĀYAN $J$. Chem.

Vol. 14 | No. 2 |974-980| April - June | 2021

chloride (201 mg,) and the mixture stirred for $30 \mathrm{~min}$ instead tosyl oxime, isolated cyclized product as spirobicyclic pyrrolidine which was purified by flash column chromatography to afford compound $\mathbf{1 2}$ (111 mg, 56\%) as a crystalline solid; m.p. $=99{ }^{\circ} \mathrm{C} ; \mathrm{R}_{f}=0.51$ (EtOAc : petrol, 1:1); $v_{\max }\left(\right.$ film) $/ \mathrm{cm}^{-1} 2934$, $1780,1732,1456,1172,915,735 ; \delta_{\mathrm{H}}\left(400 \mathrm{MHz} ; \mathrm{CDCl}_{3} ; \mathrm{Me}_{4} \mathrm{Si}\right) 0.98\left(3 \mathrm{H}, \mathrm{d}, \mathrm{CH}_{3}\right), 1.79,2.05\left(\mathrm{C}\left(\mathrm{CH}_{3}\right)_{2}\right)$, $2.16\left(3 \mathrm{H}, \mathrm{s}, \mathrm{N}=\mathrm{CCH}_{3}\right), 2.64(1 \mathrm{H}, \mathrm{m}, \mathrm{C}(4) H), 2.94(2 \mathrm{H}, \mathrm{d}, J 7.5 \mathrm{~Hz}, \mathrm{C}(3) H H) ; \delta_{\mathrm{C}}\left(100 \mathrm{MHz} ; \mathrm{CDCl}_{3}\right.$; $\left.\mathrm{Me}_{4} \mathrm{Si}\right)$ 18.2, $\left.\left(\mathrm{CH}_{3}\right), 21.5\left(\mathrm{~N}=\mathrm{CCH}_{3}\right), 28.4,28.7 \mathrm{C}\left(\mathrm{CH}_{3}\right)_{2}\right), 32.1(\mathrm{C} 4), 43.2(\mathrm{C} 3), 88.9(C 5), 106.7$ $\left.C\left(\mathrm{CH}_{3}\right)_{2}\right), 167.9(2 \times \mathrm{COO}), 182.8(\mathrm{C} 2) ; \mathrm{m} / \mathrm{z}\left(\mathrm{ESI}^{+}\right) 248\left([\mathrm{M}+\mathrm{Na}]^{+}, 95 \%\right), \mathrm{HRMS}\left(\mathrm{ESI}^{+}\right) \mathrm{C}_{11} \mathrm{H}_{15} \mathrm{NNaO}_{4}{ }^{+}$ $\left([\mathrm{M}+\mathrm{Na}]^{+}\right)$requires 248.0899 ; found 234.0839 .

The instruments used for the data analysis are NMR instruments with models are AVG400/AVF400/AVB400 and IR-Spectrometer used FTS6000/FTS7000 and for mass spectra we used LC-MS LCT

\section{RESULTS AND DISCUSSION}

Spiro-heterobicycles appear in countless natural products and clinically valuable compounds ${ }^{29}$ possibly among the most challenging structural motifs to synthesize, spirocyclic synthesis has inspired chemists for decades. Meldrum's acid appears to be an attractive reagent in organic synthesis owing to its high acidity, steric rigidity and high reactivity. ${ }^{30}$ Following the methodology developed ${ }^{27}$ using oxime ether mediated ring closure, Meldrum's acid could be very useful to synthesize spiro-heterobicyclic rings. Indeed, the spiro-heterobicycle 3 could be helpful to construct non-natural amino acid by solvolysis of Meldrum's acid with alcohols would give a diastereomeric mixture of a half acid ester; and there could be further interconverted. The half carboxylic acid can be converted into amine or carbamate, through acid azide formation and followed by Curtius rearrangement would give an isocyanate, which could be easily converted into amine or carbamate by hydrolysis or alcohol treatment. ${ }^{31}$

The required spiro-heterobicyclic compound 3 could be synthesized by following the sequence of reactions. The reaction of Meldrum's acid with methyl vinyl ketone in presence of 1.1 eq. of anhydrous $\mathrm{K}_{2} \mathrm{CO}_{3}$ and 1 eq. of benzyltriethylammonium chloride in acetonitrile as the solvent, at $50-60{ }^{\circ} \mathrm{C}$ for $8-10$ $\mathrm{hr}^{32}$ afforded the Michael adduct $\mathbf{1}$ in $81 \%$ yield with some double Michael addition product as a byproduct which was minimized by using 1.1 eq. base. The Michael adduct 1 was readily converted to the corresponding oxime using standard conditions $\left(\mathrm{NH}_{2} \mathrm{OH} . \mathrm{HCl}\right.$ and $\mathrm{Et}_{3} \mathrm{~N}$ in EtOH stirred at $50^{\circ \mathrm{C}}$ for $1 \mathrm{hr}$ ) and afforded $\mathbf{2}$ in $77 \%$ yield. The crude oxime 2 was used directly and treatment with $p$-toluenesulfonyl chloride and base (excess $\mathrm{Et}_{3} \mathrm{~N}$ in dry $\mathrm{CH}_{2} \mathrm{Cl}_{2}$ at $\mathrm{rt}$ ) gave the cyclized product as Spiro-3,4-dihydro-2Hpyrrole 3 directly in $78 \%$ of yield (Scheme-1) instead of the expected tosyl oxime (Scheme-1).<smiles>CC1(C)OC(=O)CC(=O)O1</smiles>

Meldrum's acid

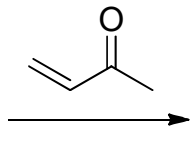

a

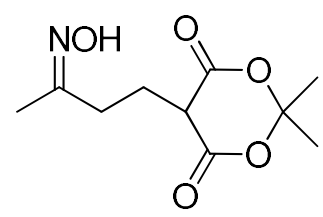

2<smiles>CC(=O)CCC1C(=O)OC(C)(C)OC1=O</smiles>

$1,81 \%$

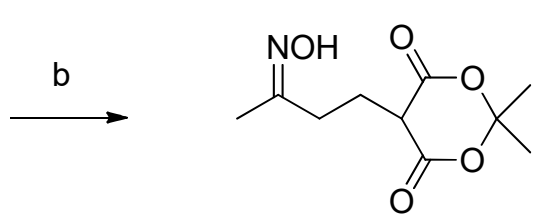

2, $77 \%$

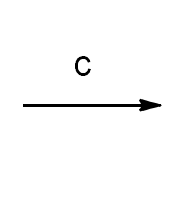

$3,78 \%$

Reagent and Conditions: (a) Anhydrous $\mathrm{K}_{2} \mathrm{CO}_{3}$ benzyltriethylammonium chloride, acetonitrile heated 50-60 ${ }^{\circ \mathrm{C}}$; (b) $\mathrm{NH}_{2} \mathrm{OH} . \mathrm{HCl}, \mathrm{Et}_{3} \mathrm{~N}, \mathrm{EtOH}$, heated 50 ${ }^{\circ \mathrm{C}}$; (c) pTsCl, $\mathrm{Et}_{3} \mathrm{~N}, \mathrm{CH}_{2} \mathrm{Cl}_{2}$, rt. Scheme-1: Synthesis of Spiro Compound

The resultant Spiro-3,4-dihydro-2H-pyrrole 3 is crystalline and this product was fully characterized by single-crystal X-ray crystallographic analysis (Fig.-2), clearly demonstrating the fully orthogonal nature of the two heterocyclic rings. The spiro-heterobicycle 3 was previously reported by Danheiser ${ }^{33}$ and he obtained it as oil but NMR data agree. 
RASĀYAN J. Chem.

Vol. 14 | No. 2 |974-980| April - June | 2021
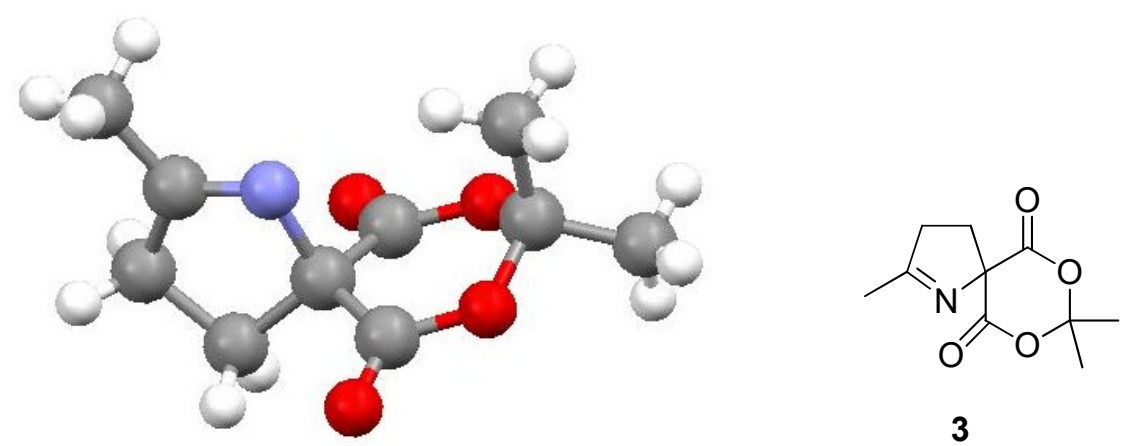

3

Fig.-2: X-ray Crystal Structure for Spiro-3,4-dihydro-2H-pyrrole 3

Similarly, the Michael addition reaction with Meldrum's acid and $\alpha, \beta$-unsaturated ketones such as 1phenylbut-2-en-1-one, 1-phenylprop-2-en-1-one, pent-3-en-2-one and chalcone were carried out in presence of anhydrous $\mathrm{K}_{2} \mathrm{CO}_{3}$ and 1 eq. of benzyltriethylammonium chloride in acetonitrile as the solvent, at $50-60{ }^{\circ} \mathrm{C}$ for $8-10 \mathrm{hr}$ except chalcone other ketone gave good yield as Michael adduct as shown in scheme 2 . The same Michael adduct on oximation using standard condition and followed by treatment with $p$-toluenesulfonyl chloride and base (excess $\mathrm{Et}_{3} \mathrm{~N}$ in dry $\mathrm{CH}_{2} \mathrm{Cl}_{2}$ at $\mathrm{rt}$ ) gave the cyclization product Spiro-3,4-dihydro-2H-pyrrole as $\mathbf{6 , 9}$ and $\mathbf{1 2}$ in good yield as shown in Scheme-2 but as the compounds are solid but the formation of single crystal was so difficult and all compounds are characterized by Mass and NMR spectroscopy. The same click chemistry was not possible with chalcone may be due to high steric factor.<smiles>CC1(C)OC(=O)CC(=O)O1</smiles>

Meldrum's acid<smiles>[R]C=CC([R])=O</smiles>

a<smiles>[R]C(=O)CC([R])C1C(=O)OC(C)(C)OC1=O</smiles>

$\mathrm{R}=\mathrm{Me}, \mathrm{R}^{\prime}=\mathrm{Ph} 4 \quad 67 \%$

$\mathrm{R}=\mathrm{H}, \quad \mathrm{R}^{\prime}=\mathrm{Ph} 753 \%$

$\mathrm{R}=\mathrm{Me}, \mathrm{R}=\mathrm{Me} 1076 \%$

$\mathrm{R}=\mathrm{Ph}, \mathrm{R}^{\prime}=\mathrm{Ph} 13$ didn't work<smiles>[R]C(=[NH2+])CC([R])C1C(=O)OC(C)(C)OC1=O</smiles>

$\mathrm{R}=\mathrm{Me}, \mathrm{R}^{\prime}=\mathrm{Ph} 5 \mathbf{7 8 \%}$

$\mathrm{R}=\mathrm{H}, \quad \mathrm{R}=\mathrm{Ph} 8 \mathrm{81 \%}$

$\mathrm{R}=\mathrm{Me}, \mathrm{R}^{\prime}=\mathrm{Me} 1179 \%$ $\mathrm{R}=\mathrm{Ph}, \mathrm{R}^{\prime}=\mathrm{Ph}$<smiles>[R]C(=N)CC([R])C1C(=O)OC(C)(C)OC1=O</smiles>

$\mathrm{R}=\mathrm{Me}, \mathrm{R}^{\prime}=\mathrm{Ph} 5 \mathbf{7 8 \%}$

$\mathrm{R}=\mathrm{H}, \quad \mathrm{R}^{\prime}=\mathrm{Ph} 8 \mathrm{81 \%}$

$\mathrm{R}=\mathrm{Me}, \mathrm{R}^{\prime}=\mathrm{Me} 1179 \%$

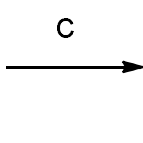

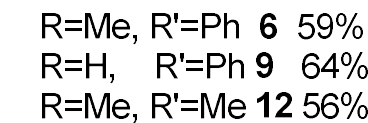

Reagent and conditions: (a) Anhydrous $\mathrm{K}_{2} \mathrm{CO}_{3}$ benzyltriethylammonium chloride, acetonitrile heated 50-60 ${ }^{\circ \mathrm{C}}$; (b) $\mathrm{NH}_{2} \mathrm{OH} . \mathrm{HCl}, \mathrm{Et}_{3} \mathrm{~N}$, EtOH, heated 50 $0^{\circ \mathrm{C}}$; (c) pTsCl, $\mathrm{Et}_{3} \mathrm{~N}, \mathrm{CH}_{2} \mathrm{Cl}_{2}, \mathrm{rt}$. Scheme-2: Synthesis of Substituted Spiro Compounds ${ }^{36}$

\section{CONCLUSION}

In summary, we have developed a one-pot methodology for the synthesis of spiro-heterobicyclic compounds as Spiro-3,4-dihydro-2H-pyrroles with alkyl and aryl substitutions on the five-member heterocycle. It was also concluded that both aryl groups as substitution were not possible as we have started with chalcone as starting material and reaction did not work. The method is useful to synthesize the rigid spiro-heterocycle which can also be converted to the non-natural amino acids by hydrolysis to half acid ester and interconvert to amino group thought Curtius rearrangement reaction. This methodology developed will be very useful to get quick access to synthesize Spiro-3,4-dihydro-2H-pyrroles which is a part of many natural products of high importance. 
RASĀYAN J. Chem.

Vol. 14 | No. 2 |974-980| April - June | 2021

\section{ACKNOWLEDGEMENT}

I am very much thankful to Prof. Mark Moloney Oxford University, the UK for their kind support. I also extend my gratitude to my Management PES and principal and my family for continuous help in my research.

\section{REFERENCES}

1. A. R. Katritzky, C. A. Ramsden, E. F. V. Scriven, R. J. K. Taylor, G. Jones, Edition PergamonElsevier: Oxford, pp.1-388 (2008)

2. D. L. Quin, J. A. Tyrell, Fundamentals of Heterocyclic Chemistry; Wiley: New Jersey, pp. 222-225 (2010), DOI:10.1021/acs.joc.0c00109

3. J. Alvaroz-Builla, J. Vaquero, Barluenga, Edition Wiley-VCH: Weinheim, pp.269-375 (2011)

4. B. A. Trofimov, A. I. Mikhaleva, E. Y. Schmidt, L. N. Sobenina, Chemistry of Pyrroles; CRC Press: Boca Raton, (2015)

5. G. W. Gribble, J. A. Joule, Edition Elsevier Oxford, pp.165 (2016)

6. E. A. Vessally, RSC Advances, 6, 18619 (2016), DOI:10.1039/C5RA20706A

7. N. Zhou, H. T. Zhu, D. S. Yanga, Z. H. Guan, Organic Biomolecular Chemistry, 14, 7136(2016), DOI:10.1039/C6OB00962J

8. A. Sharma, P. Piplani, Journal of Heterocyclic Chemistry, 54(1), 27(2017), DOI:10.1002/jhet.2550

9. V. Bhardwaj, D. Gumber, V. Abbot, S. Dhiman, P. Sharma, RSC Advances, 5, 15233(2015), DOI: $10.1039 / \mathrm{c} 4 \mathrm{ra} 15710 \mathrm{a}$

10. R. Khajuria, S. Dham, K. K. Kapoor, RSC Advances, 6, 37039(2016), DOI:10.1039/c6ra03411j

11. M. S. Mohamed, R. H. A. El-Hameed, A. Sayed, Journal of Advanced Pharmaceutical Research, 1(1), 1(2017), DOI:10.21608/APRH.2017.16155

12. R. Kaur, V. Rani, V. Abbot, Y. Kapoor, D. Konar, K. Kumar, Journal of Pharmaceutical Chemistry and Chemical Science, 1, 17(2017)

13. M. R. Kumar, A. Manikandan, A. Sivakumar, V. V. Dhayabaran, Bioorganic Chemistry, 81, 44 (2018), DOI:10.1016/j.bioorg.2018.07.037

14. M. Aldeghi, S. Malhotra, D. L. Selwood, A.W.E. Chan, Chemical Biology and Drug Design, 83(4), 450 (2014), DOI: 10.1111/cbdd. 12260

15. T. J. Richie, S. J. Macdonald, Drug Discovery Today, 14(21), 1011(2009), DOI: 10.1016/j.drudis.2009.07.014

16. Y. Zheng, C. M. Tice, S.B. Singh, Bioorganic Medical Chemistry Letters, 24(16), 3673(2014), DOI: 10.1016/j.bmcl.2014.06.081

17. Santos, Tetrahedron, 70(552), 9735(2014), DOI:10.1016/j.tet.2014.08.005

18. Gubba Reddy, Gopal Reddy, M. Durgaprasad, M. P. Bhadra, Organic Biomolecule Chemistry, 13, 8729(2015), DOI: 10.1039/C5OB01077B

19. E. J. Lewis, L. G. Hunsicker, W. R. Clarke, T. Berl, M. A. Pohl, J. B. Lewis, E. Ritz, R. C. Atkins, R. Rohde, I. Raz, New England Journal of Medicine, 345, 851(2001), DOI:10.1056/NEJMoa011303

20. B. M. Massie, P. E. Carson, J. J. McMurray, M. Komajda, R. McKelvie, M. R. Zile, S. Anderson, M. Donovan, E. Iverson, C. Staiger, New England Journal of Medicine., 359, 2456(2008), DOI: 10.1056/NEJMoa0805450

21. H. Kuhl, Climacteric, 8, 3(2005), DOI:10.1080/13697130500148875

22. H. Wiesinger, M. Berse, S. Klein, S. Schwend, J. Hochel, F. S. Zollmann, Journal of Clinical Pharmacology, 80, 1399(2015), DOI:10.1111/bcp.12745

23. M. Claire, H. Faraj, G. Grassy, A. Aumelas, A. Rondot, G. Auzou, Journal of Medicinal Chemistry, 36, 2404(1993), DOI:10.1021/jm00068a018

24. A. J. Friedman, Cutis., 96, 216(2015), DOI:10.2147/CCID.S137794

25. J. Bariwal, L. G. Voskressensky, Van der Eycken, Chemical Society Review, 47, 3831(2018), DOI: 10.1039/C8OB03122C

26. A. Iwata, S. Inuki, S. Oishi, N. Fujii, H. Ohno, Tetrahedron,71, 6580(2015), DOI:10.3987/COM-19$\mathrm{S}(\mathrm{F}) 40$

27. Nandkishor Chandan, Amber L. Thompson and Mark G. Moloney, Organic Biomolecule Chemistry, 10, 7863(2012), DOI:10.1039/c2ob26423d 
RASĀYAN J. Chem.

Vol. 14 | No. 2 |974-980| April - June | 2021

28. T. D. Wahyuningsih, N. Kumar, D. Black, Tetrahedron, 62, 6343(2006)

29. M. Sannigrahi, Tetrahedron, 55, 9007(1999), DOI:10.1016/S0040-4020(99)00482-2

30. B. C. Chen, Heterocycles, 32, 529(1991), DOI:10.3987/REV-90-422

31. J. Sapi, J. Y. Laronze, Archive for Organic Chemistry, 7, 208(2004), DOI: $10.3998 /$ ark.5550190.0005.717

32. H. Tomori, K. Shibutani, K. Ogura, Synthesis, 3, 224(1984), DOI:10.1055/s-1984-30779

33. A. R. Renslo, R. L. Danheiser, Journal of Organic Chemistry, 63, 7840(1998), DOI: $10.1021 / \mathrm{jo} 026258 \mathrm{k}$

34. P. A. Bonvallet, E. M. Todd, Y. S. Kim, R. J. McMahon, Journal of Organic Chemistry, 67, 9031(2002), DOI: 10.1021/jo020304z

35. V. Singh, S. Kanojiya, S. Batra, Tetrahedron, 62, 10100(2006), DOI:10.1016/j.tet.2006.08.045

36. Nandkishor Chandan, Rasayan Journal of Chemistry, 13(4), 2352(2020), DOI: 10.31788/RJC.2020.1345745

[RJC-6151/2020] 